\title{
Correction for mapping errors in non-null test of aspheric surface
}

W. Weibo

Z. Mengqian

Y. Siwen

F. Zhigang

L. Jian

liujian@hit.edu.cn
Institute of Ultra-precision Optoelectronic Instrument Engineering, Harbin Institute of Technology, Harbin, 150001, China

Postdoctoral Research Station of Optical Engineering, Harbin Institute of Technology, Harbin, 150001, China

Institute of Ultra-precision Optoelectronic Instrument Engineering, Harbin Institute of Technology, Harbin, 150001, China

Institute of Ultra-precision Optoelectronic Instrument Engineering, Harbin Institute of Technology, Harbin, 150001, China

Postdoctoral Research Station of Optical Engineering, Harbin Institute of Technology, Harbin, 150001, China

Institute of Ultra-precision Optoelectronic Instrument Engineering, Harbin Institute of Technology, Harbin, 150001, China

The non-null testing methods have the potential to allow measurement of aspheric surfaces with large departures from a reference sphere. In a non-null configuration, the ray will return along a different path and interfere with a different reference ray. This difference in "mapping" between the test and reference rays creates an additional optical path difference (OPD) contribution, which causes test part errors to be mapped to the wrong location. To correct the mapping errors in non-null test of aspheric surface, correction method are proposed by ray trace and wave-front analysis. Experiments are carried out to illustrate the effectiveness of this approach. The methods can work well, despite large deviation between the theoretic value of the aspheric and the reference wave-front.

[DOI: http://dx.doi.org/10.2971/jeos.2015.15032]

Keywords: Non-null testing, aspheric surface, mapping error, ray trace

\section{INTRODUCTION}

Traditional interferometry is done in a null or near-null condition. The rays from the test part follow exactly the same path through the optical system as the reference rays and no systematic errors are induced. This null condition indicates the shape of the measured object is nearly identical to that of the reference and small deviations from the null are linearly proportional to the differences between them. Thus, it is possible to assess the shape of a measured surface using the map of differences simply by rescaling this map to relevant units. However, when testing an asphere without a null, the rays from the test wavefront and reference wavefront will not trace the same optical path. This results in aberrations due to the optical properties of the optical components and the departure from the reference sphere not canceling each other, and thus will be present in the final measurements. Such aberrations, called retrace errors, lead to wide difference between the obtained surface figures and depend on the optical layout of the instrument which can be significant [1]-[3].

The induced aberrations can be classified into phase errors and mapping errors. Mapping error occurs under conditions of nonlinear magnification: the wave front from the test surface becomes stretched or squeezed at various positions, which causes test part errors to be mapped to the wrong loca- tion. Eliminating the lens distortion achieves a linear mapping in a null test, but this need not be true in a non-null test $[4,5]$.

In the case of measurements of aspheric surfaces with a spherical reference, rays from different pupil regions follow different optical paths through the system, varying with apertures and dependent on the test part, and lead to considerable testing error that cannot be negligible [6]-[8]. Especially for the sub-aperture stitching test of aspheric surface, the mapping is crucial for stitching into a unified map, when placing the sub-aperture data onto a global coordinate plane. If this effect is not corrected, the ill-conditioned character of the stitching process means that, even though the distortion may appear to be a minor factor within each sub-aperture, significant errors can be introduced to the stitched data.

In this paper, we use the simple ray tracing method to analyze the mapping error in non-null aspheric testing. Based on the analysis of retrace error and wave-front distortion, effective methods are proposed to correct the errors in non-null aspheric testing. The methods work well, despite large deviation between the theoretic value of the aspheric and the reference wave-front. Experiments are carried out to illustrate the effectiveness of this approach. 


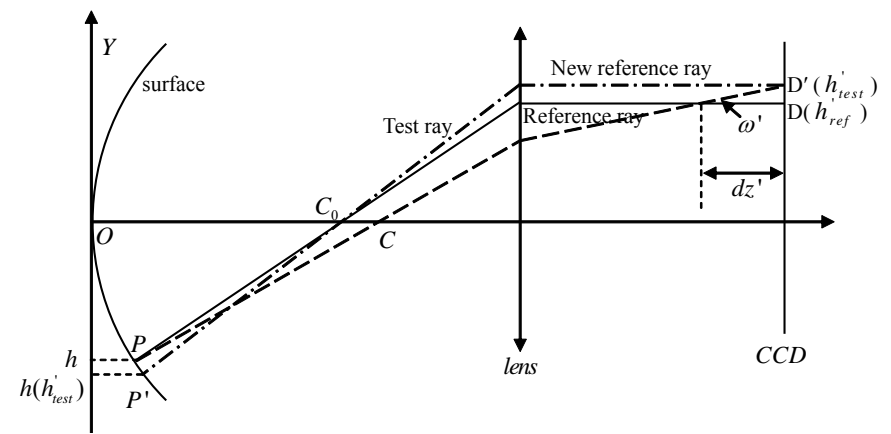

FIC. 1 Ray trace in non-null test of aspheric surface.

\section{MAPPING ERROR ANALYSIS OF NON-NULL ASPHERIC TESTING}

In the non-null test of aspheric surface, the local test surface is not normal to the incident ray. The ray will return along a different path and interfere with a different reference ray, as shown in Figure 1. This difference in "mapping" between the test and reference rays in the viewing system creates an additional OPD contribution that depends upon both the shape and misalignment of the test surface. This mapping-induced OPD error can be thought of as a shear of the test wave-front and reference wave-front in the exit pupil of the viewing system $[9,10]$.

A mapping error occurs for a test ray which intersects the image plane at a location different from that predicted by the first order magnification of the system. For a spherical reference wave-front, the mapping error is a nonlinear magnification, which appears as distortion in the test wave-front. The complete mapping functions are [5]

$$
\begin{aligned}
h_{\text {test }}^{\prime}(h) & =m h+e_{y}\left[r_{\text {test }}(h), h\right] \\
h_{r e f}^{\prime}(h) & =m h+e_{y}[0, h]
\end{aligned}
$$

Only linear magnification $m$ and distortion aberration $e_{y}[0, h]$, determine the mapping in a null test. As the test rays $r_{\text {test }}(h)$ becomes larger, all other transverse ray aberrations affect the test ray mapping.

In the null test, the common path situation implies $r_{\text {test }}=r_{\text {ref }}$, so $e_{y}\left[r_{\text {test }}(h), h\right]-e_{y}\left[r_{r e f}(h), h\right] \approx 0$, there is no mapping error. In the non-null test, as shown in Figure 1, the original reference and test rays do not intersect at the detector plane. The mapping error causes test part errors to be mapped to the wrong location.

This mapping is not at 1:1 from test surface to detector pixel coordinates. Instead, the mapping can be simply written as

$$
\rho^{\prime}=(1+\varepsilon) \rho
$$

The radial coordinate of the ideal wave-front is denoted $\rho$, and that of the distortion wavefront $\rho^{\prime}$.

An effective step therefore is to express $\varepsilon\left(\rho^{2}\right)$ as a polynomial

$$
\varepsilon\left(\rho^{2}\right)=\varepsilon_{0}+\varepsilon_{1} \rho^{2}+\varepsilon_{2} \rho^{4}+\ldots+\varepsilon_{n} \rho^{2 n}
$$

Where in the dimensionless constants in the expression evidently satisfy $\left|\varepsilon_{i}\right| \ll 1$ for all $i$. $\varepsilon_{0}$ gives a fine correction to the pixel scale, $\varepsilon_{1}$ corrects for what is usually called third-order distortion, $\varepsilon_{2}$ for fifth-order, etc. Since the low-order terms generally dominate, it is often sufficient for $n$ to be one or two.

As mentioned above, the mapping error creates an additional OPD contribution that depends upon the misalignment, such as defocus, and the departure of the test aspheric surface from the reference sphere.

If a known focus shift is made in the optical test, we have a wave-front change that is expressed as the wave-front form for defocus is shown in

$$
W(\rho, \theta)=k \rho^{2}
$$

where $k$ is the defocus in waves of error at the edge of the aperture.

According to Eq. (4) and Eq. (5), for $n=1$, Eq. (4) can be expressed as

$$
\rho_{i}=\rho_{m}\left(1+\varepsilon \rho_{m}^{2}\right)=\rho_{m}+\varepsilon_{m}^{3}
$$

where $\varepsilon$ is the fractional distortion at the edge of the aperture. The error was introduced into the position coordinate $\rho$ by third order distortion.

Substituting Eq. (7) into Eq. (6) the form of the wave-front at the detector can be derived as

$$
W(\rho, \theta)=k\left(\rho^{2}+2 \varepsilon \rho^{4}+\varepsilon^{2} \rho^{6}\right)
$$

The measured error can be expressed as

$$
\Delta W(\rho, \theta)=k\left(2 \epsilon \rho^{4}+\varepsilon^{2} \rho^{6}\right)
$$

When alignment errors and higher order terms in this wavefront expression are ignored, the measured error can be expressed as

$$
\Delta W(\rho, \theta)=2 k \varepsilon \rho^{4}
$$

We can see that a known amount of focus in the wave-front contributes spherical aberration to the measured result. This defocus will lead to considerable testing error that cannot be negligible. The departure of the test aspheric surface from the reference sphere can result in similar consequences.

For mapping error correction, the relation between the ideal wavefront and distortion wavefront can be written as

$$
W(\rho)=W_{d}(\rho)=W_{i}[(1+\varepsilon) \rho]
$$

The wavefront error caused by mapping error as follows

$$
\begin{aligned}
\Delta W & =W_{d}(\rho)-W_{i}(\rho) \\
& =W\left(\frac{\rho}{1+\varepsilon}\right)-W(\rho) \\
& \approx W[(1-\varepsilon) \rho]-W(\rho)
\end{aligned}
$$

The error of radial coordinate can be written as

$$
\Delta \rho=\varepsilon \rho
$$

Then

$$
\frac{\partial W}{\partial \rho}=\frac{W[(1-\varepsilon) \rho]-W(\rho)}{\varepsilon \rho}
$$




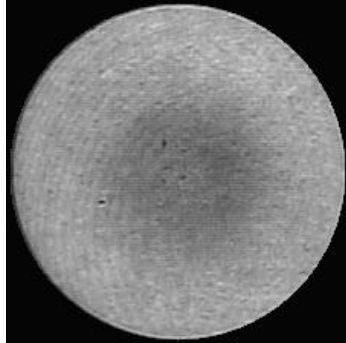

(a)

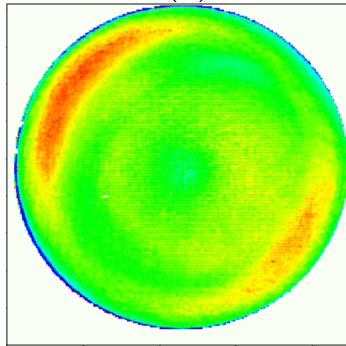

(c)

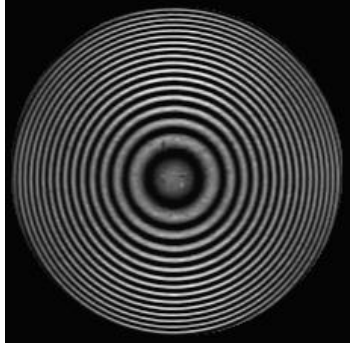

(b)

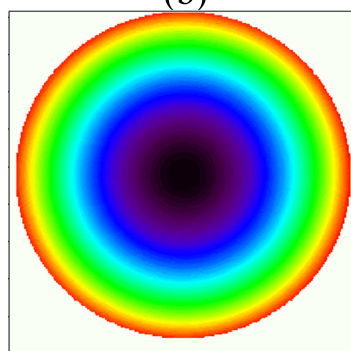

(d)
FIG. 2 Measurements taken at null and non-null positions.

So we can get

$$
\Delta W=\varepsilon \rho \frac{\partial W}{\partial \rho}
$$

If we take $n=1$, the compensators can be written in polynomial as

$$
\begin{aligned}
\Delta W(x, y)= & \varepsilon_{0}\left[x \frac{\partial f(x, y)}{\partial x}+y \frac{\partial f(x, y)}{\partial y}\right] \\
& +\varepsilon_{1}\left[x \frac{\partial f(x, y)}{\partial x}+y \frac{\partial f(x, y)}{\partial y}\right] \rho^{2}
\end{aligned}
$$

The form of the compensators presented above depends on the measured aperture data. By controlling wavefront focus and monitoring the resulting aberration, an estimate of the system distortion can be made. Once the distortion is determined, a polynomial transformation can be applied to remap the surface data appropriately.

\section{EXPERIMENT}

\subsection{Measurements of spherical surfaces in non-null condition}

The effectiveness of this approach is illustrated by example. For that purpose we used a calibrated spherical surface accurate to within $\lambda / 10$ and an F/1.5 transmission sphere. A defocused sphere was used to generate large departures from a reference sphere in a non-null configuration.The calibrated spherical surface was measured in a non-null condition (defocused position) resulting in high density fringes in order to compare these results with measurements obtained in the null condition (residual focus was removed in all measurements).

Figure 2 shows measurements taken at different positions. The calibrated spherical surface was moved from the null position to non-null position by translating it along the optical axis of the interferometer.

Figure 3 and Figure 4 plot results of the aberration coefficients of Zernike polynomials. Figure 3 shows the aberrations calcu-

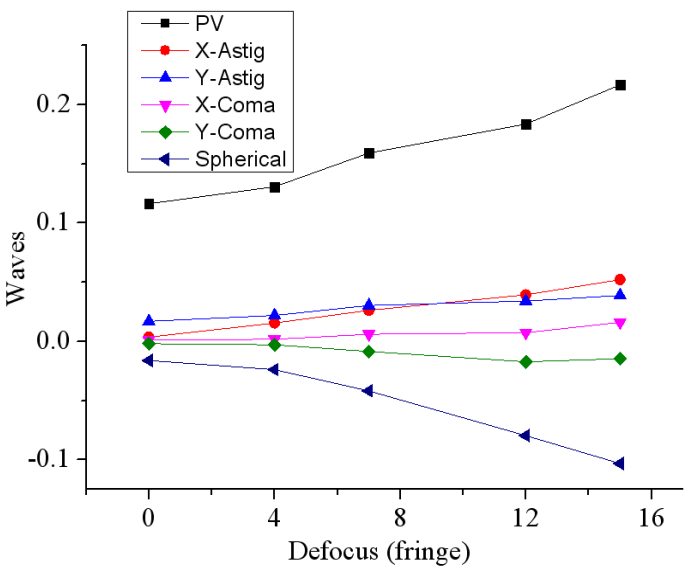

FIG. 3 Aberrations with no corrections and power removed.

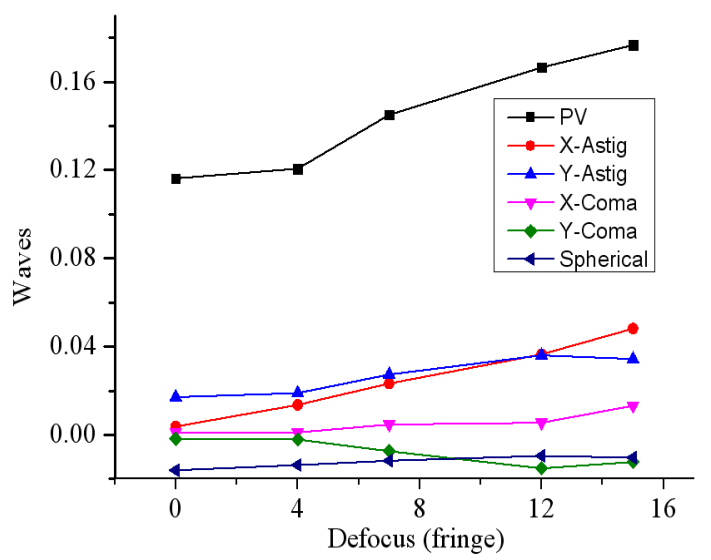

FIG. 4 Aberrations with mapping error corrections and power removed.

lated for the phase map obtained directly from the interferograms, while Figure 4 shows the aberration results after the "mapping error" correction. From these results it is clear that the error is dominated by spherical aberration as a function of focus position in the optical test. These data can be used to write a set of equations relating wavefront coefficients for spherical (Z9) and focus (Z4) aberrations for individual measurement cases of varying defocus. This set of equations can then be used to calculate wave-front coefficients in order to estimate the system distortion. The performance of the mapping error correction is a function of fringe density, and is better than $\lambda / 20$ at 20 fringes.

\subsection{Measurements of aspheric surfaces in non-null condition}

Measurement of aspheric surface has been taken using the same procedure as the one described for the measurement of the calibrated spherical surface. The interferograms were recorded at the location where the fringe density was minimal. The radius of curvature (ROC) of the illuminating beam at this location corresponds to the best fit sphere of the aspheric surface. This ROC value is used in addition to the measurement and ray trace results in order to generate the actual surface shape. The measured surface shape is then subtracted from the actual lens design prescription to create a difference phase map. Figure 5 is an example of an aspheric measure- 


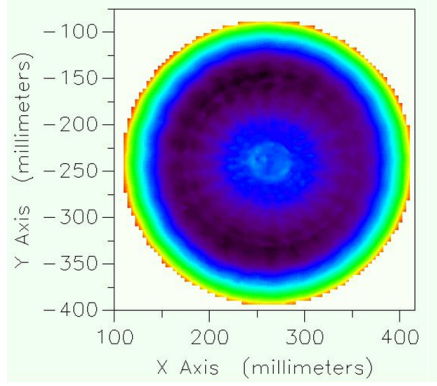

(a) $\mathrm{PV}=0.3551 \lambda, \mathrm{RMS}=0.0416 \lambda$

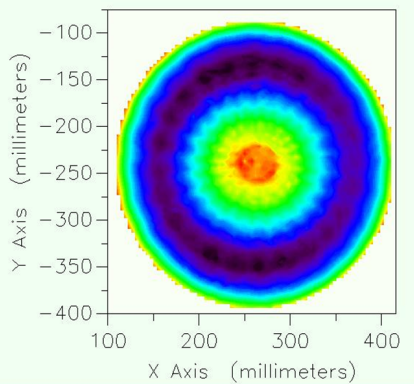

(b) $\mathrm{PV}=0.2637 \lambda, \mathrm{RMS}=0.0339 \lambda$
FIG. 5 An uncorrected measured aspheric wave-front (left), the corrected wave-front minus the design prescription (right).

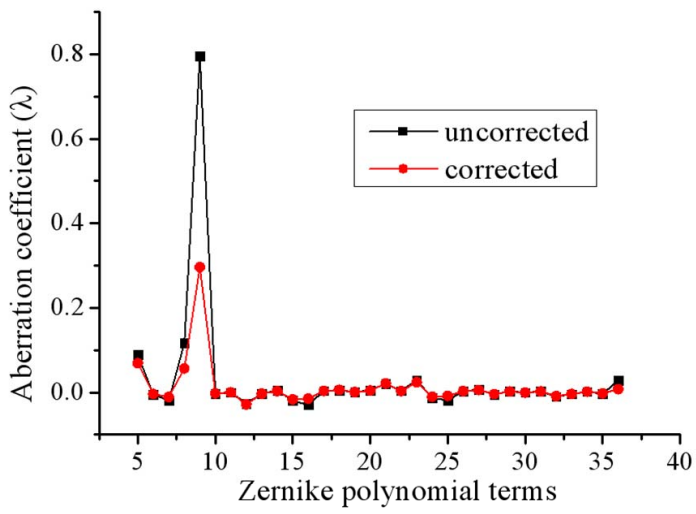

FIG. 6 A comparison with a standard measurement device.

ment in computing the final result. Figure 6 is a comparison with a standard measurement device. The aberration results after the "mapping error" correction shows the effectiveness of the proposed method.

\section{CONCLUSION}

The mapping error of non-null aspheric testing is analyzed based on the ray tracing and wavefront analysis. The effectiveness of correcting methods proposed is illustrated by the examples of rotationally symmetric surfaces such as spherical surface and aspheric surface in nonnull condition. These methods can improve the capability of interferometer for taking measurements in non-null conditions, has been demonstrated to produce quality measurements of aspheric surfaces and provide accurate knowledge of the position of surface features in order to efficiently fabricate aspheric optical surfaces.

\section{ACKNOWLEDGEMENTS}

This research is supported by Natural Science Foundation of China (51205089 51275121 and 51475111), China Postdoctoral science foundation (2012M520726), Heilongjiang Postdoctoral Fund (LBH-Z12128).

\section{References}

[1] P.r Szwaykowski, and R. Castonguay, "Measurements of aspheric surfaces," Proc. SPIE 7063, 706317 (2008)

[2] A. E. Lowman, and J. E Greivenkamp, "Interferometer induced wavefront errors when testing in a non-null configuration," Proc. SPIE 2004, 173-181 (1993).

[3] R. 0. Gappinger, and J. E Greivenkamp, "Iterative reverse optimization procedure for calibration of aspheric wavefront measurements on a non-null interferometer," Appl. Optics 43, 5152-5161 (2004).

[4] J. Pfund, N. Lindlein, and J. Schwider, "Nonnull testing of rotationally symmetric aspheres: a systematic error assessment," Appl. Optics 40(4), 439-446(2001).

[5] P. E. Murphy, T. G. Brown, and D. T. Moore, "Interference Imaging for Aspheric Surface Testing," Appl. Optics 39, 2122-2129 (2000).

[6] D. Liu, Y. Yang, and C. Tian, "Practical methods for retrace error correction in nonnull aspheric testing," Opt. Express 17, 7029-7035 (2009).

[7] R. Jozwicki, "Influence of aberrations of Fizeau interferometer elements on measurement errors," Appl. Optics 30(22), 3126-3132 (1991).

[8] N. Gardner, and A. Davies, "Retrace error evaluation on a figuremeasuring interferometer," Proc. SPIE 5869, 1-8 (2005).

[9] Q. Xin, Y. Gao, and Y. Zeng, "An analysis of the fundamental measuring error of aspherical surface by sheering interferometer," Proc. SPIE 2536, 148-153 (1995).

[10] B. E. Truax, "Absolute interferometric testing of spherical surfaces," Proc. SPIE 1400, 61-68 (1991). 